\title{
Rectal cancer presenting as Fusobacterium nucleatum pyogenic liver abscess: A case report
}

\author{
Tovey M, Rizvi FA, Raman S, Wheatley KE
}

\begin{abstract}
Introduction: Fusobacterium nucleatum (F. nucleatum) is a non-spore forming, anaerobic, gram-negative bacteria usually associated with periodontal disease and rarely reported in association with pyogenic liver abscess. Although common symptoms of colonic cancer are well known, unusual infections may herald presence of such a cancer. Streptococcus bovis bacteraemia is an accepted diagnostic marker for occult colonic malignancy. Case Report: We report a 72-year-old Caucasian male patient who presented to us as an emergency with $F$. nucleatum hepatic abscess and was found to have a rectal cancer on further gastrointestinal screening. Literature search suggests no previous direct association of $F$. nucleatum with colorectal cancer. Conclusion: The hepatic abscess was successfully treated with percutaneous drainage and intravenous antibiotics followed by definitive surgery for his cancer at a later stage. A brief review of
\end{abstract}

Tovey $\mathrm{M}^{1}$, Rizvi $\mathrm{FA}^{2}$, Raman $\mathrm{S}^{3}$, Wheatley $\mathrm{KE}^{4}$, Affiliations: ${ }^{1}$ Foundation Year 1 Doctor, Sandwell General Hospital, West Bromwich, UK; ${ }^{2}$ Core Training (Surgery), Birmingham, West Midlands Deanery, Birmingham, UK; ${ }^{3}$ Specialist Registrar (General Surgery), Birmingham, West Midlands Deanery, UK; ${ }^{4}$ Consultant Surgeon, Sandwell and West Bromwich Hospitals NHS Trust, Birmingham, UK.

Corresponding Author: Dr. Rizvi FA; Core Training (Surgery), Birmingham, West Midlands Deanery, Birmingham, UK; Phone: +44-7950112889; Email: fahadrizvi@doctors.org.uk

Received: 22 December 2010

Accepted: 27 February 2011

Published: 30 June 2011 literature of association of pyogenic liver abscess and occult colonic cancer is presented.

Keywords: Fusobacterium nucleatum, Pyogenic liver abscess, Colon cancer, Sigmoid cancer

$* * * * * * * * *$

Tovey M, Rizvi FA, Raman S, Wheatley KE. Rectal cancer presenting as Fusobacterium nucleatum pyogenic liver abscess: A case report. International Journal of Case Reports and Images 2011;2(6):16-19.

$* * * * * * * * *$

doi:10.5348/ijcri-2011-06-39-CR-4

\section{INTRODUCTION}

Pyogenic liver abscess is a serious and potentially life-threatening condition, with an incidence between $0.006 \%-2.2 \%$ of hospital admissions and mortality reported to range from $10-40 \%$ [1].

The most commonly isolated organisms associated with pyogenic liver abscesses are Klebsiella pneumonia and Escherichia coli [2]. Rarely, Fusobacterium nucleatum has been implicated with hepatic abscess though never previously in association with sigmoid carcinoma. It has been reported once following a colonoscopic biopsy for ulcerative colitis [3].

Fusobacteria nucleatum is an anaerobic gramnegative, non-spore forming bacilli found in normal flora of the oral cavity. It is one of the most common oral species isolated from extra-oral infections such as blood, brain, lung, liver, joints, abdominal, obstetrical and gynaecological infections and abscesses. It has recently been reported as a cause of liver abscess in a patient suffering from recurrent periodontal disease [4]. 
We report the first case of $F$. nucleatum pyogenic liver abscess as the initial presentation of a rectosigmoid carcinoma.

\section{CASE REPORT}

A 72-year-old gentleman presented with a three week history of fatigue, night sweats and rigors. He was initially assessed by his General Practitioner who started a course of penicillin but, unfortunately, on completion of the antibiotics the rigors recurred. There was no associated cough, diarrhoea, weight loss, abdominal pain, dysuria or contact with any tuberculosis patients.

Past medical history was remarkable only for nasal polyps and hypertension, for which he was treated with ramipril and bendroflumethiazide. The patient reported alcoholic intake of 16 units per week, with no history of intravenous drug abuse or of receiving any blood or blood product transfusions. The patient was immunocompetent and not diabetic or on steroid treatment.

Examination was unremarkable but initial blood tests revealed an elevated ALP 421 IU/L (normal range 20-130iu/L), CRP $180 \mathrm{mg} / \mathrm{L}$ (Normal range $<5 \mathrm{mg} / \mathrm{L}$ ) and white cell count $16.1 \times 10^{9} / \mathrm{L}$ (normal range $4.0-$ 11.0x109/L) with a neutrophilia of $15.40 \times 10^{9} / \mathrm{L}$ (normal range 1.70-7.50 x109/L). Ultrasound scanning of the abdomen revealed three echo poor areas in the liver, the largest of which was $3.8 \mathrm{~cm} \mathrm{x} 4.2 \mathrm{~cm}$. This was thought to represent either an abscess or a metastatic deposit.

Blood culture did not yield any results, but the patient was given a stat dose of $320 \mathrm{mg}$ of gentamicin and $1.2 \mathrm{gm}$ intra-venous co-amoxiclav three times a day. A CT scan of the abdomen was performed which revealed a hepatic abscess of $3.8 \times 4.2 \mathrm{~cm}$ (Figure 1), which was drained percutaneously under

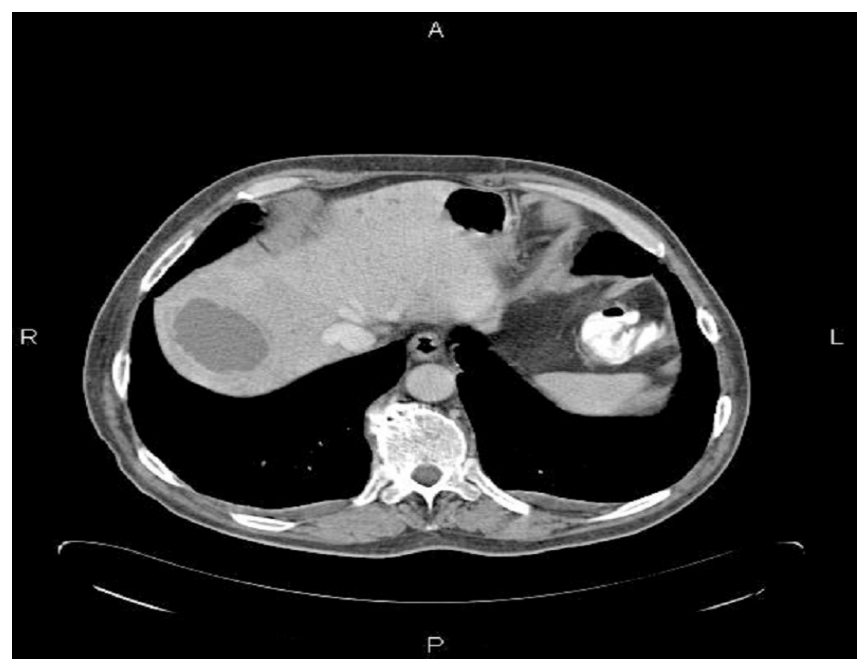

Figure 1: CT scan showing liver abscess, which was drained percutaneously. ultrasound guidance. This aspirate was cultured and grew $F$. nucleatum noted to be sensitive to penicillin and metronidazole. Follow-up CT scan was performed, which showed marked resolution of the hepatic abscess.

The initial CT scan was also noted to show an abnormal mass at the recto-sigmoid junction (Figure 2). This was discussed at a subsequent colo-rectal multi-disciplinary meeting and thought to be a rectosigmoid tumor. The patient underwent colonoscopy, which revealed a large, friable, polypoid lesion extending from the mid- to upper-rectum, which was reported as a poorly differentiated adenocarcinoma on biopsy.

Anterior resection was decided to be the definitive treatment once the hepatic abscess fully resolved, and was successfully undertaken several weeks later. The patient recovered well from his surgery and was discharged home with subsequent follow up.

\section{DISCUSSION}

Colorectal cancer is often associated with abscessformation in the ischio-rectum, abdominal wall, psoas and paravertebral areas. It may through direct extension of the tumor or as a result of bacteraemia from colonic pathogens. There is an increasing association between colonic cancer and liver abscess over the last few years. It has been reported that a pyogenic liver abscess may be associated with, or be the initial presentation of an underlying rectosigmoid or colonic neoplasm [5-7].

The most common organism found in such liver abscess is Streptococcus bovis and also that this bacteraemia may precede the diagnosis of the cancer by several years [8]. It is now well established that $S$. bovis bacteraemia is associated with gastrointestinal

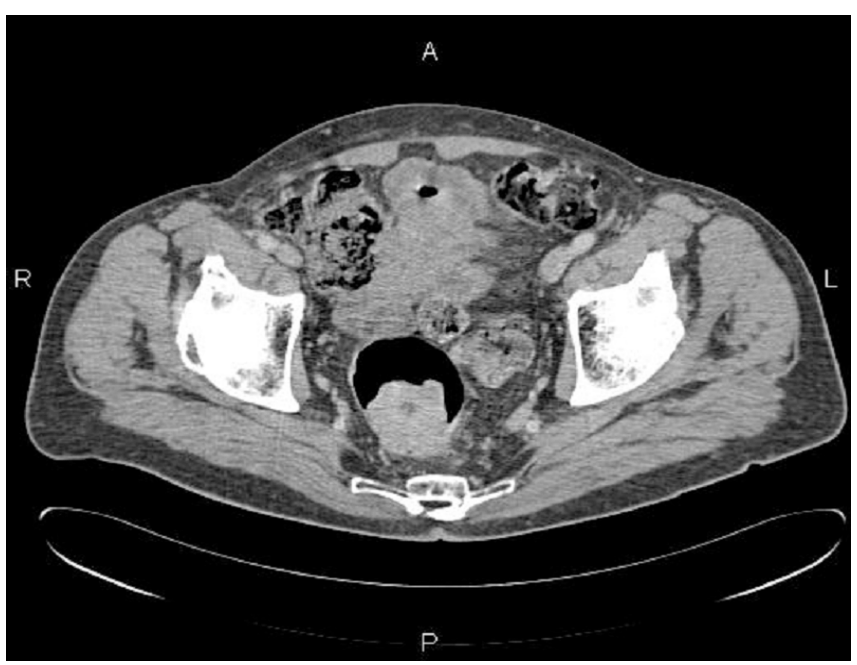

Figure 2: CT scan of the same patient showing high rectal cancer. 
disease, particularly colonic neoplasia. It has also been suggested that extracolonic malignancy is a common feature of a large proportion of patients with $S$ bovis Bacteremia [9].

Liver abscesses caused by periodontal bacterial infection are rare, however, the incidence is expected to increase in the future as periodontitis is extremely common and is on the rise as one of the most common chronic infections in the world [10]. Clinically significant infections caused by members of the genus Fusobacterium are rare, affect distinct patient groups and are associated with good clinical outcomes in the majority of cases [11].

Fusobacterium bacteraemia is uncommon, accounting for approximately $0.9 \%$ of patients with bacteraemia and is associated with a high mortality rate for patients with renal insufficiency, heart failure or malignancy [12]. The proposed mechanism for bacteraemia is following mucosal disruption by the neoplasm resulting in the underlying blood vessels becoming exposed to the faecal flora [13].

$F$. nucleatum has previously been implicated in pyogenic liver abscess formation,14 and in one study Fusobacterium species were implicated in $4.3 \%$ of microbiological isolates [15].

F. nucleatum is a non-sporulating, immotile, Gramnegative bacterium which has pathogenic potential due to its frequency and numbers found in periodontal lesions. Interestingly, of all the bacteria linked with periodontal disease, $F$ nucleatum has coaggregration properties which allow it to transport periodonto pathogenic bacteria [16]. This is imperative in supporting our hypothesis that $F$. nucleatum may have breached the mucosa of the colon due to the carcinoma, leading to a bacteraemia resulting in a hepatic abscess.

There was no sign of periodontal disease in our patient, the bacteria may have originated from colon, which makes this report a unique case presentation of Fusobacterium nucleatum presenting as a liver abscess in association with colorectal cancer.

\section{CONCLUSION}

We are reporting the first incidence in literature of colonic cancer in association with Fusobacterium nucleatum. Also, there is also no evidence to suggest $F$. nucleatum has been associated with colonic cancer in isolation.

It is our aim to reinforce the suggestion that on exclusion of the predominant liver abscess etiologies, a diagnosis of colonic carcinoma is considered. It is also our view that unusual bacteraemia and microbiological isolates should be thoroughly investigated despite possible occurrence at sites distal to their usual colonization.

\section{Author Contributions}

Tovey M. - Conception and design, Acquisition of data, Analysis and interpretation of data, Critical revision of the article, Final approval of the version to be published

Rizvi F. A. - Conception and design, Acquisition of data, Analysis and interpretation of data, Critical revision of the article, Final approval of the version to be published

Raman S. - Conception and design, Acquisition of data, Analysis and interpretation of data, Critical revision of the article, Final approval of the version to be published

Wheatley K. E. - Conception and design, Acquisition of data, Analysis and interpretation of data, Critical revision of the article, Final approval of the version to be published

\section{Guarantor}

The corresponding author is the guarantor of submission.

\section{Conflict of Interest}

The authors declare that they have no competing interests.

\section{Copyright}

(C) M. Tovey et al. 2010; This article is distributed under the terms of Creative Commons attribution 3.0 License which permits unrestricted use, distribution and reproduction in any means provided the original authors and original publisher are properly credited. (Please see www.ijcasereportsandimages.com /copyright-policy.php for more information.)

\section{REFERENCES}

1. Huang, C. J., H A Pitt, P A Lipsett, F A Osterman, Jr, K D Lillemoe, J L Cameron, and G D Zuidema. Pyogenic hepatic abscess. Changing trends over 42 years. Ann Surg. 1996;223(5): 600-9.

2. Chen SC, Wu WY, Yeh CH, Lai KC, Cheng KS, Jeng LB, Wang PH, Lin DB, Chen CC, Lee MC, Bell WR. Comparison of Escherichia coli and Klebsiella pneumoniae liver abscesses. Am J Med Sci. 2007;334(2):97-105.

3. Wells CD, Balan V, Smilack JD. Pyogenic liver abscess after colonoscopy in a patient with ulcerative colitis. Clin Gastroenterol Hepatol. 2005;Dec;3(12):xxiv.

4. Kim YH, Yoon HJ, Park CW, Kim JH, Lee MK, Kim KB, Na DJ, Kim JM. A Case of Liver Abscess Caused by Fusobacterium nucleatum in a Patient with Recurrent Periodontal Diseases. Korean J Gastroenterol. 2011 Jan 25;57(1):42-6.

5. Hsu WH, Yu FJ, Chuang CH, Chen CF, Lee CT, Lu CY. Occult colon cancer in a patient with diabetes and recurrent Klebsiella pneumoniae liver abscess. Kaohsiung J Med Sci. 2009;Feb;25(2):98-103.

6. Giuliani A, Caporale A, Demoro M, Scimò M, Galati F, Galati G. Silent colon carcinoma presenting as a 
hepatic abscess. Tumori. 2007 Nov-Dec;93(6):6168.

7. Yokota T, Iwamoto K, Watanabe Y, Yamauchi H, Kikuchi S, Hatori M. Pyogenic liver abscesses secondary to carcinoma of the sigmoid colon: a case report and clinical features of 20 cases in Japan. Ups J Med Sci. 2005;110(3):241-4.

8. Honberg O.Z., Gutschick E. Streptococcus bovis bacteremia and its association with alimentary-tract neoplasm. Lancet. 1987;1(8525):163-4.

9. Jason S. Gold, MD; Sancar Bayar, MD; Ronald R. Salem, MD. Association of Streptococcus bovis Bacteremia With Colonic Neoplasia and Extracolonic Malignancy. Arch Surg. 2004;139:7605 .

10. Yoneda $\mathrm{M}$ et al. Liver abscess caused by periodontal bacterial infection with Fusobacterium necrophorum. Hepatol Res. 2011;41(2):194-6.

11. P. Huggan, D. Murdoch. Fusobacterial infections: Clinical spectrum and incidence of invasive disease. Journal of Infection. 2008;57(4):283-9.

12. $\mathrm{Su} \mathrm{CP}$ et al. Fusobacterium bacteremia: clinical significance and outcomes. J Microbiol Immunol Infect. 2009;42(4):336-42.

13. Siegert CEH, Overbosch D. Carcinoma of the colon presenting as Streptococcus sanguis bacteremia. Am J Gastroenterol. 1995;90:1528-9.

14. Tweedy CR, White WB. Multiple Fusobacterium nucleatum liver abscesses. Association with a persistent abnormality in humoral immune function. J Clin Gastroenterol. 1987;9(2):194-7.

15. Lok KH, Li KF, Szeto ML. Pyogenic liver abscess clinical profile, microbiological characteristics, and management in a Hong Kong hospital. J Microbiol Immunol Infect. 2008;41(6):483-90.

16. Signat B, Roques C, Poulet P, Duffaut D. Fusobacterium nucleatum in Periodontal Health and Disease. Curr Issues Mol Biol. 2011;13(2):2536. 